\title{
Mediación biopedagógica de salud y vida, casos de enfermedad cardiovascular
}

\author{
Health and Life's Biopedagogical Mediation, Cases of Cardiovascular Disease
}

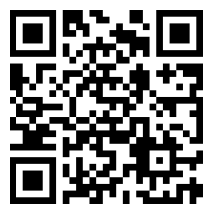

\author{
Jorge Salas-Cabrera' \\ Universidad Nacional \\ Heredia, Costa Rica \\ jorge.salas.cabrera@gmail.com \\ http://orcid.org/0000-0002-9226-3144
}

Recibido 18 de marzo de 2016 • Corregido 14 de noviembre de 2016 • Aceptado 10 de abril de 2017

Resumen: Esta investigación tuvo como propósito analizar la aplicación de una mediación biopedagógica basada en el constructivismo, el aprendizaje y la comprensión de hábitos de vida saludables (alimentación, actividad física, relaciones sociales, estado psico-afectivo e información sobre la enfermedad cardiaca) de pacientes de rehabilitación cardiaca. Se utilizó el enfoque cualitativo de naturaleza ideográfica, que analiza comportamientos, hábitos y costumbres de las personas de forma individual; apreciándolos en toda su riqueza; para comprender razones y decisiones. En el estudio participaron cinco pacientes (4 hombres y 1 mujer), quienes tuvieron un evento cardiovascular, en edades entre 40 y 65 años, la mediación biopedagógica por paciente tuvo una duración de 6 semanas con 18 sesiones, para efectos de la recolección de datos la totalidad de participantes sabía leer y escribir. Se identificaron cinco categorías de análisis: emocional-afectiva, nutricional, social, comportamiento-cognitivo y actividad física. Para la obtención de las expresiones pertinentes, para el análisis respectivo se utilizaron tres instrumentos: relatos de vida, el instrumento de frases incompletas y, finalmente, el instrumento de libre asociación. Como conclusión de este estudio se logró mejorar la comprensión de este grupo de pacientes acerca de la importancia de modificar hábitos de vida, específicamente en las cinco categorías de análisis, lo cual se evidenció en el seguimiento que se les realizó dos años después de haber recibido la mediación biopedagógica.

Palabras claves: Rehabilitación; paciente cardiaco; constructivismo; mediación pedagógica.

\footnotetext{
1 Profesor de la Escuela Ciencias del Movimiento Humano y Calidad de Vida desde hace 12 años, en la cátedra de Fisiología del ejercicio y prescripción del ejercicio, así, como de los cursos del entrenamiento contra resistencia. Coordinador del proyecto de rehabilitación cardiaca fase Ill e investigador en las áreas de fisiología del ejercicio, pedagogía y salud integral.
} 
doi: http://dx.doi.org/10.15359/ree.21-2.1

URL: http://www.una.ac.cr/educare

CORREO: educare@una.cr

\begin{abstract}
This research aimed to analyze the application of a bio-pedagogical mediation built on the basis of constructivism in the learning and understanding of healthy living habits, specifically those related to food, physical activity, social relations, psycho-affective status and information about heart disease in patients undergoing cardiac rehabilitation. A qualitative approach to the ideographic nature was implemented; it analyzed individual behaviors, habits, and customs, appreciating them in all their richness, to understand the reasons and decisions. The study involved five patients (4 men and 1 woman), who had cardiovascular events, aged between 40 to 65 years old. The bio-pedagogical mediation lasted 6 weeks, and 18 sessions of one hour per patient were held; each patient was able to read and write with no problems. Five categories of analysis were conducted: emotional-affective, nutritional, social, cognitive behavior and physical activity. To obtain the relevant expressions, three instruments were used for the respective analysis: life stories, incomplete sentences, and free association. The conclusion of this study was the improvement of the patient's understanding of the importance of changing life habits, specifically in the five categories of analysis; this conclusion was verified in the follow-up done to the participants for two years after receiving the bio-pedagogical mediation.
\end{abstract}

Keywords: Rehabilitation; heart patient; constructivism; pedagogical mediation.

Durante muchos años se ha considerado que la ciencia es provisional, pues a partir de ella se crean teorías sujetas al cambio en función de nuevos hallazgos, a partir de los cuales, se renuevan los antiguos paradigmas. Las teorías de la educación no escapan a este fenómeno, y las investigaciones en diferentes áreas educativas han permitido incrementar el conocimiento y facilitar intervenciones pedagógicas para un mejor aprendizaje. Estos avances ofrecen los principios y el sustento teórico para mejorar los procesos metodológicos educativos durante la enseñanza y, además, los pone a disposición para cuando se requiera en la práctica pedagógica (Palacios, 2000; Ruiz, 2007; Torres, 2008). Por lo tanto, toda mediación pedagógica ofrece diferentes propuestas teóricas y metodológicas que permiten desarrollar diversas interacciones con el estudiantado. En el caso del enfoque constructivista, el uso de herramientas metodológicas es primordial para orientar a quienes están en el proceso de transformación de la propia realidad; y también; influye en las representaciones que tienen de ella. La persona es capaz de hacer una reconstrucción según su comprensión, que a la vez le evidencia su propia situación; posteriormente y de forma gradual romperá con viejos hábitos de vida o maneras de actuar a través de conexiones entre los viejos y nuevos conocimientos, situación que facilita la elaboración de un nuevo esquema mental (Bravo, 2002; Vygotski, 1978).

En el diseño de una mediación pedagógica basada en la propia construcción del aprendiente existen varios modelos pedagógicos de corte constructivista, dentro de los cuales se encuentran: el modelo constructivista cognitivo (el cual fundamenta su base teórica en la psicología y la epistemología genética de Piaget), el modelo constructivista sociocultural (que se refiere al constructivismo social o socioconstructivismo, basado en las ideas y planteamientos de Vygotsky), el modelo constructivista social (el cual postula que el sujeto aprendiz dentro de 
su mundo cognitivo regula su proceso de aprendizaje entre lo cognitivo y las relaciones sociales) y finalmente el modelo constructivista radical (que postula que el conocimiento, sin importar cómo se defina, está en la mente de las personas y el sujeto que aprende no tiene otra alternativa que construir lo que conoce sobre la base de su propia experiencia) (Serrano y Pons, 2011; Soler, 2006). Con los aportes de las diferentes teorías constructivistas, la ejecución de una mediación pedagógica debe desarrollarse a la luz de experiencias en las cuales la persona transforme la manera de aprender, para que pueda diseñar y construir procesos de aprendizaje, por sí misma, convirtiendo el nuevo conocimiento en algo significativo, y que le permita un cambio en su esquema mental y, posteriormente, un cambio en la manera de comportarse (Hernández, 2008).

Estos elementos tienen un importante alcance en la realidad costarricense, cuando se analizan en función de la presencia de enfermedades cardiacas, pues, según la Caja Costarricense de Seguro Social, se presenta una mayor incidencia de ellas en personas de edades entre los 40 y los 80 años. De acuerdo con las teorías educativas en personas adultas; quienes se encuentran en estas edades cuentan con un bagaje cognitivo particular y una personalidad definida; por lo que requieren procesos de formación específicos; entre ellos el desarrollo de procesos educativos que tengan un aporte positivo tanto en la rehabilitación física como en la modificación de los hábitos de vida, con la estrategia fundamental de que las personas estén activas en la construcción de su propio conocimiento, a partir del análisis crítico y la reflexión sobre su propio comportamiento y sus experiencias de vida (Cubero 2009; Kunoichisama, s. f.).

Es así como el constructivismo describe los principios básicos del aprendizaje, concibiendo a la persona adulta como participante activa, apoyada en mediaciones pedagógicas, las cuales permiten el establecimiento de relaciones entre su vivencia y la nueva información, reestructurando y re-organizando su pensamiento o estructuras cognitivas, para atribuirle un significado a lo nuevo. A partir de esto, se considera fundamental desarrollar, en la persona adulta con enfermedad cardiaca, las estrategias de comprensión necesarias para la metarreflexión (analizar, observar, reinterpretar, reflexionar) de las circunstancias que la llevaron a presentar dicha patología y de las estrategias que le faciliten enfrentar su recuperación.

Esta comprensión se considera significativa en la medida en que: genere un mejor desempeño de la persona, mejore la auto-identificación de necesidades, aumente su capacidad de concientizar el significado de la enfermedad cardiaca, y reconozca el origen y las posibles modificaciones de los hábitos de vida, con el fin de alcanzar una exitosa recuperación. Es decir, una mediación "biopedagógica" debe facilitar a la persona la identificación de aquellos aspectos que generaron el desarrollo de la enfermedad y, con base en ello, iniciar el proceso de construcción del nuevo conocimiento con las herramientas aplicadas durante la mediación (Núñez, Solano, González-Pienda y Rosário, 2006; Perera, 2009). 
doi: http://dx.doi.org/10.15359/ree.21-2.1

URL: http://www.una.ac.cr/educare

CORREO: educare@una.cr

Según Bravo (2002), en el enfoque constructivista el uso de herramientas metodológicas orienta, a quien aprende, a transformar su propia realidad y también las representaciones que tiene de ella, con la posibilidad de llevar el nuevo conocimiento a un nivel significativo, que mediante los procesos cognitivos y metacognitivos, facilite la resolución de problemas de diversa índole (Hernández, 2008).

\section{Referente teórico}

De acuerdo con la visión empirista filosófica, un modelo es el resultado del proceso de generar una representación abstracta, conceptual, gráfica o visual para analizar, describir, explicar, simular y predecir fenómenos o procesos (Gallego y Ongallo, 2004).

De esta forma se elaboran los modelos pedagógicos teóricos, que deben construirse sobre teorías que faciliten, confronten y relacionen propiedades de la realidad, para tratar de proveer la enseñanza de recursos útiles y significativos para el aprendizaje; de no ser así, es posible que un modelo pedagógico tenga poca pertinencia y sea fácilmente debatible. (Cerezo, 2008; Gallego y Ongallo, 2004; lbáñez, 2007).

El modelo constructivista cuyos enunciados operan con la idea de que el sujeto que aprende toma la responsabilidad de su propio proceso de conocimiento con la colaboración y mediación de un personal tutor, incentiva a cada persona a: plantear preguntas, hacer ligámenes entre el viejo y el nuevo conocimiento, reflexionar sobre sus experiencias previas y visualizar nuevos conocimientos en su proceso de rehabilitación (Castro, 2004; Cerezo, 2008; Hernández 2007). Para lograrlo, el aprendizaje debe ser activo y debe estimular a las personas a participar en actividades propias de su aprendizaje, en lugar de que solamente se dediquen a observar de manera pasiva lo que su docente o personal tutor explica (Hernández, 2007).

En el marco del modelo constructivista, se trata de aprender haciendo " "aprender a aprender"; entre sus objetivos también está la necesidad de estimular las habilidades de comprensión de los individuos con el fin de que progresen y evolucionen secuencialmente en las estructuras cognitivas y que accedan a nuevos conocimientos cada vez más elaborados; lo cual depende de dos aspectos básicos: la representación que se tiene de la nueva información y la actividad interna (o externa) que se desarrolle respecto del proceso de aprendizaje (Cerezo, 2008; Hernández, 2007). Algunos de los elementos que propone el modelo constructivista son: impulsar la autonomía e iniciativa de la persona; utilizar herramientas interactivas y manipulables; usar conceptos cognitivos como analizar, predecir, crear, deducir, estimar, elaborar y pensar, y utilizar la comprensión de conceptos que tenga la persona, antes de compartir con ella su propia interpretación.

Estos elementos deben estar presentes en cualquier proceso de conocimiento, para que las personas aprendan diferentes maneras de enfrentar el mismo problema. No se trata de enseñar "cosas acabadas", sino más bien, se debe orientar para que descubran por sí mismas posibles 
soluciones (Cerezo, 2008). Por esto, la mediación es una intervención que se practica para enriquecer la relación de la persona con su ambiente (Cuenca, 2007); es decir, durante el desarrollo de una mediación pedagógica es adecuado presentarle al sujeto que aprende una variedad de situaciones significativas y formas de proceder ante alguna situación de la vida real, de manera que sea capaz de resolverla por sí. Esto facilita la adquisición de conocimientos significativos.

La mediación "biopedagógica" en el modelo constructivista propone que el mediador tenga claridad de la intencionalidad o metas de los participantes, en este caso de las personas con enfermedad cardiaca, a través de la buena comunicación, para alcanzar un aprendizaje efectivo. Debe tomar en cuenta todas las experiencias previas del sujeto paciente, su historia personal y no dejar de lado que para esta población el "aquí y ahora" es un imperativo. Por lo tanto, es fundamental involucrarse emocionalmente y disponerse para cumplir los objetivos planteados (Cabrera, Motta, Rodríguez y Velázquez, 2009).

La mediación debe favorecer los sentimientos de competencia y logro, para que la persona se sienta capaz y reconozca que este proceso es muy importante para alcanzar el éxito. Esto asegura una adecuada disposición para el aprendizaje y para la aceptación de nuevos retos, lo cual genera confianza y autoeficacia al emprender las tareas. El reconocimiento de los logros y las habilidades para realizar la actividad con éxito mejora y facilita la capacidad para tomar decisiones y para cooperar con otras personas (Cabrera et al., 2009).

En este sentido, la mediación pedagógica debe tener como referencia métodos que permitan construir un modelo eficaz en el aprendizaje de cualquier contenido, entre ellos está el que propone el aprendizaje por descubrimiento. Este aprendizaje se desarrolla cuando su docente le facilita al estudiantado las herramientas necesarias para que descubra por sí solo lo que desea aprender, y permite que la persona se apropie de su proceso y busque información, la seleccione, la organice e intente resolver, con ella, diferentes problemáticas que enfrenta.

De acuerdo con Bruner (1988, p. 21): “...El método por descubrimiento, permite al individuo desarrollar habilidades en la solución de problemas, ejercitar el pensamiento crítico, discriminar lo importante de lo que no lo es, preparándolo para enfrentar los problemas de la vida...." En este caso el sujeto docente organiza problemas o situaciones problemáticas, sugiere fuentes de información y está presto a colaborar con las necesidades de su aprendiz; esto permite que la persona construya hábitos y sea rigorosa en los procesos comportamentales, para descubrir nuevos conocimientos; sin embargo, tiene mayor facilidad de aprendizaje una vez que el contenido ha sido asimilado (Restrepo 2005).

Así mismo, según Bruner, Olver y Greenfield (1966), descubrir y generar un pensamiento crítico que permita enfrentar las situaciones de la vida depende de que el aprendizaje sea significativo, por lo cual, en el proceso de rehabilitación cardiaca, el paciente debe descubrir su propio camino en la recuperación. En la mayoría de las investigaciones realizadas en 
doi: http://dx.doi.org/10.15359/ree.21-2.1

URL: http://www.una.ac.cr/educare

CORREO: educare@una.cr

rehabilitación cardiaca sistematizada, el sujeto paciente se encuentra bajo la supervisión de especialistas que dirigen los procesos de recuperación, por lo cual no existe una participación activa, ni se presentan estrategias que permitan que la persona descubra por si misma el nuevo conocimiento. Estas situaciones limitan la capacidad de descubrir (Cossette, Frasure-Smith, Lespérance, 2001; Del Río, Turro, Mesa, Mesa y Lorente, 2005).

Por su parte, el aprendizaje de solución de problemas permite y facilita que la persona avance hacia un nuevo estado de comprensión, resuelva problemas y reflexione sobre ellos; también permite auto-evaluar y auto-organizar el nuevo conocimiento a través de las mediaciones pedagógicas, en donde su participación se desarrolla de una forma activa, construyendo soluciones y recursos estratégicos idóneos para adquirir conocimiento. Según Coronel y Curotto (2008, p. 464), "La enseñanza desde esta perspectiva pretende poner el acento en actividades que plantean situaciones problemáticas cuya resolución requiere analizar, descubrir, elaborar hipótesis, confrontar, reflexionar, argumentar y comunicar ideas".

Esquivias, González y Muria (2003) manifiestan que el aprendizaje de solución de problemas es la forma más elaborada de aprendizaje, debido a que la persona define nuevas ideas, además determina que cuando existe un problema, se requiere del conocimiento acerca de qué lo genera; es decir, comprender la situación y además, tener la capacidad para hacerle frente; esto permite la transferencia de lo aprendido. Al ser la persona capaz de resolverlo, se habilita para adaptarse al medio y modificarlo de manera parcial.

Para fomentar el aprendizaje mediante la solución de problemas, es indispensable que la persona desarrolle habilidades de pensamiento, como, por ejemplo, representación y comprensión. Al articularlas con símbolos y conceptos, se orienta la creación de formas de pensamiento que permiten solucionar un problema determinado (Esquivias et al., 2003).

Para fines prácticos, en esta investigación el sujeto paciente cardiaco, al explorar sobre la causa del problema, debe reflexionar acerca de las posibles soluciones que le permitan mejorar su condición; desde esta perspectiva, es claro que dichas soluciones tienen que estar apegadas a su realidad, al contexto en el cual se desenvuelve, para que realmente puedan ser significativas y aplicadas en la rutina diaria de su vida, para ello requiere una intensa actividad constructiva que implica desplegar los procesos cognitivos (Serrano y Pons 2011). Es, entonces, en este caso, responsabilidad del propio sujeto paciente generar su propio aprendizaje de manera que le sea lo suficientemente significativo para modificar comportamiento y, a la vez, hábitos de vida.

Es entonces importante utilizar la teoría del aprendizaje autónomo; de acuerdo con Escobar (2003), para una persona ser autónoma implica la capacidad de poder fijar sus normas y elegir por sí misma las reglas que va a respetar; mediante el aprendizaje autónomo la persona logra ser capaz de manejarse con autonomía en diversas y variadas situaciones, propias del constructivismo. Esta teoría es determinante para la recuperación de la persona con enfermedad 
cardiaca, pues requiere la capacidad de dirigirse, no solo en aspectos de rehabilitación, sino también, en lo que se refiere a la prevención del mal. Además, permite entender que el proceso de rehabilitación va más allá de mejorar la parte orgánica, pues envuelve lo emocional, lo psicológico, lo espiritual y lo social, es decir, constituye una manera integral. Desde el punto de vista ontológico, el ser humano es un ente que, a raíz de la motivación por aprender, logra más conocimiento, lo que al fin y al cabo llega a ser significativo, no solo en un área específica, sino en todo aquello que le cause motivación.

En ese sentido, el fundamento del aprendizaje autónomo determina que la persona o paciente ante todo tenga la disponibilidad para aprender; en este caso, porque en la mediación pedagógica constructivista se van a obtener insumos que generan reflexiones críticas sobre la situación; además, estos promueven cambios en el estilo de vida, comprensión, rescate del nuevo conocimiento y descarte de aquello que fue, en algún momento, una falsa creencia acerca de la enfermedad, de manera que le ayude a confrontar, de forma constructiva, su patología y su proceso de rehabilitación.

Según lo señala Villavicencio (2004, p. 2) “...Se alcanza la autonomía cuando la persona llega a ser capaz de pensar por sí misma con sentido crítico, teniendo en cuenta muchos puntos de vista, tanto en el ámbito moral como en el intelectual. Mientras que la autonomía moral trata sobre lo 'bueno' o lo 'malo'; lo intelectual trata con lo 'falso' o lo 'verdadero'". Al hablar de rehabilitación, lo adecuado es que el sujeto paciente, por su autonomía, tenga un cambio de comportamiento que impacte de manera positiva e integral su vida; es decir, en lo psicofisiológico, en lo social y en lo espiritual.

Para lograr la evolución positiva en la recuperación, es ideal que autoevalúe los cambios que va experimentando y vaya construyendo, con el apoyo de la mediación pedagógica, las estrategias que le sirvan para modificar las conductas poco saludables, el aspecto fundamental en el marco del aprendizaje autónomo (Villavicencio, 2004).

Ello tiene la finalidad de buscar el aprendizaje significativo caracterizado por la interacción entre las ideas principales existentes en la estructura cognitiva y la nueva información, con lo cual permite una reestructuración cognitiva, lo que contribuye a una diferenciación, elaboración y estabilidad de los conocimientos previos existentes (Sánchez, Moreira y Caballero, 2011), ya que, si los conocimientos previos existentes en la estructura cognitiva son claros, estables y diferenciables, facilitan la resolución de problemas.

El aprendizaje significativo surge cuando la persona, como ser que construye su propio conocimiento, tiene la posibilidad de relacionar conceptos al darles sentido a partir del conocimiento que ya posee, de manera que el aprendizaje se da cuando los contenidos están relacionados con algún aspecto existente en la vida de la persona o, en este caso particular, del paciente cardiaco (Sosa-Simón, Pavia-Carrillo y Mendoza-Cruz, 2009). En ese sentido, Ausubel, Novak y Hanesian (1983) manifiestan que el aprendizaje del alumnado depende, en gran 
doi: http://dx.doi.org/10.15359/ree.21-2.1

URL: http://www.una.ac.cr/educare

CORREO: educare@una.cr

medida, de la estructura cognitiva previa, la cual se relaciona con la nueva información y se genera una "estructura cognitiva", en donde se encuentra un conjunto de conceptos, ideas que un individuo posee en un determinado campo del conocimiento, así como su organización.

Como se puede apreciar y de acuerdo con lo expresado por Ausubel et al. (1983), en la medida en que la persona haga relaciones acerca de lo que aprende con su estructura cognitiva previa permite alcanzar un aprendizaje que le es significativo y que será recordado a lo largo de los años. Al depender de cada estructura cognitiva de la persona, el tiempo en que se adquiera el conocimiento aprendido estará sujeto a la capacidad que tenga cada una en hacerlo significativo y la forma de relacionarlo con las ideas y conceptos previos. Es importante que a la persona el nuevo conocimiento alcanzado con respecto a sus causas y consecuencias, formas de cuidado y recuperación, le signifique y logre modificar el comportamiento con respecto a la enfermedad.

El conocimiento no es más una simple representación de la realidad externa; es el resultado de la interacción entre el sujeto que aprende (cognitivamente) y sus "prácticas sensoriales". El sujeto adulto que aprende abandona la típica pasividad y construye nuevas experiencias, participa activamente en el proceso de forma real y verdadera. De acuerdo con Moreno (2010, p. 5) “... La educación para adultos se percibe como un proceso permanente e integral de formación y actualización, dirigido a la preparación de una persona crítica, democrática, dinámica, pero sobre todo esto, elevar su autoestima haciéndolo sentir productivo".

Es importante recordarque por las características deesta investigación y con lacomprensión de que los problemas cardiovasculares atacan mayormente a personas adultas, se enfatizó en la aplicación del constructivismo a través de una mediación pedagógica dirigida a la educación adulta; es por ello que, de acuerdo con lo que manifiesta Moreno (2010), la educación de la persona adulta con enfermedad cardiovascular debe ser un proceso permanente, en donde se integren los aspectos idóneos para su rehabilitación, tal es el caso de la alimentación, el ejercicio, el manejo emocional-afectivo y las relaciones sociales, que le permitan convertirse en una persona activa y crítica en la construcción de nuevo conocimiento. El aprendizaje es un proceso de autoconstrucción de conocimiento a través de la vida, lo que lleva al auto-direccionamiento y perfeccionamiento continuo.

Finalmente, se encuentra el medio ambiente, que está determinado por tres tipos: el medio ambiente inmediato, el cual es creado para realizar la actividad educativa o el aprendizaje; el segundo tipo de ambiente está determinado por la organización educativa, en la cual se facilitan los recursos materiales y humanos; y el tercero, las agrupaciones e instituciones sociales (Salinas, Huertas, Porras, Amador y Ramos 2006). Los procesos educativos son claves en las intervenciones preventivas en el ámbito de salud, particularmente aquellos que han evolucionado de una relación emisor-receptor, a una comunicación en la que el sujeto profesional de la salud comparte sus conocimientos; por lo tanto, quien está en actividad de recepción pasa de una actitud pasiva a una activa y responsable. 
Es decir, que la persona tiene la intención de recibir ese conocimiento y poder llevarlo a la práctica, tal como lo señala la teoría de la acción razonada y la teoría de la planificación de la conducta. Sin embargo, muchas de las intervenciones educativas en pacientes con enfermedad cardiovascular se han basado en metodologías conductistas (Hernández y Arranz, 2009; Mora, 2009).

En conclusión, la manera para tener éxito en la labor de aprendizaje en la persona adulta depende no solo de profundizar en la actividad, sino también de darle la misma importancia a los aspectos externos que la rodean. Es valioso, desde el constructivismo, que la persona participe en su propio aprendizaje, que proponga estrategias que le sirvan de apoyo en su recuperación y para organizarse en el diario vivir, esto genera un impacto en el aprendizaje significativo y, por consiguiente, un cambio de comportamiento hacia una mejor calidad de vida.

\section{Metodología}

Es una investigación cualitativa ideográfica, ya que no se trata de generalizar resultados a toda una población, las conclusiones aplican a los sujetos con quienes se trabajó, quienes expresaron sus ideas, comportamientos, hábitos y costumbres, por medio de los distintos instrumentos utilizados.

\section{Categorías y subcategorías de análisis}

Las categorías permiten derivar el contenido de reflexión y metarreflexión de cada paciente sobre su propia situación ontológica de manera constructivista. Estas categorías surgen a partir de una variedad de factores fundamentales de modificar para alcanzar una adecuada rehabilitación cardiaca. Muchas investigaciones acerca de los beneficios de la rehabilitación cardiaca argumentan la importancia en la mejora de pacientes a partir de la modificación de algunos indicadores en las áreas física, psicológica, emocional, afectiva, nutricional, educativa, sexual, social y espiritual (Álvarez, De la Rosa, Acosta, Albar y García, 2005; Del Río et al., 2005).

Por ello, para efectos de esta investigación, se llevó a cabo una intervención pedagógica desde el enfoque constructivista, de manera que el sujeto paciente, a través de la construcción de su propio conocimiento, aprendiera lo que se debe hacer desde las áreas emocionalafectiva, nutricional, física y social, para lograr una adecuada recuperación, además de un mejor conocimiento acerca de la enfermedad cardiovascular.

Las subcategorías de análisis surgen del primer contacto que tuvo el investigador con cada paciente. Se establecieron, en cada categoría, aquellos conceptos que tuvieran similitud en las conversaciones sostenidas con los sujetos de la investigación; a modo de ejemplo para el caso de la categoría emocional-afectiva, en los cinco relatos de vida se expresaron aspectos relacionados con la ira, depresión, estrés, enojo y ansiedad, por lo que el investigador decidió establecerlas 
doi: http://dx.doi.org/10.15359/ree.21-2.1

URL: http://www.una.ac.cr/educare

CORREO: educare@una.cr

como subcategorías para extraer la información más importante acerca de las creencias, experiencias, conocimientos previos que tenía cada persona con respecto a ello y, durante la mediación pedagógica, observar el avance logrado con el conocimiento que iba construyendo.

En cuanto a lo propuesto por el constructivismo, es importante abordar a cada persona según sus conocimientos, experiencias e ideas previas, de manera que le sea más significativo el nuevo conocimiento que va construyendo, por ello se establecieron las subcategorías de análisis.

\section{Fases de estrategia, abordaje y procedimiento}

El trabajo en el campo constó de tres sesiones semanales, durante 5 semanas, para un total de 15 sesiones por paciente (Figura 1), las cuales se realizaron en el programa de rehabilitación cardiaca de la Escuela Ciencias del Movimiento Humano y Calidad de Vida, de la Universidad Nacional. En la semana previa a la intervención, se contactó a 5 personas ( 4 hombres y 1 mujer) con problemas cardiovasculares; se llevó a cabo una explicación del proceso a seguir; se les invitó a firmar el consentimiento informado, si accedían a participar, el cual certificó su decisión y se les solicitaron datos socio-demográficos.

Durante 5 semanas, estas personas estuvieron en contacto con el mediador pedagógico (investigador), y además, en la intervención debieron llevar y realizar tareas que implicaban análisis y autorreflexión, a partir de material alusivo a la enfermedad cardiaca, antecedentes psicosocioafectivos y comportamentales, así como posibles estrategias para coadyuvar en el proceso de rehabilitación en sus casas. Todo lo anterior, con el fin de construir el nuevo conocimiento para una comprensión más completa de la patología y, en consecuencia, de su rehabilitación.

La síntesis del proceso de mediación expuesto anteriormente, se presenta en la Figura 1:
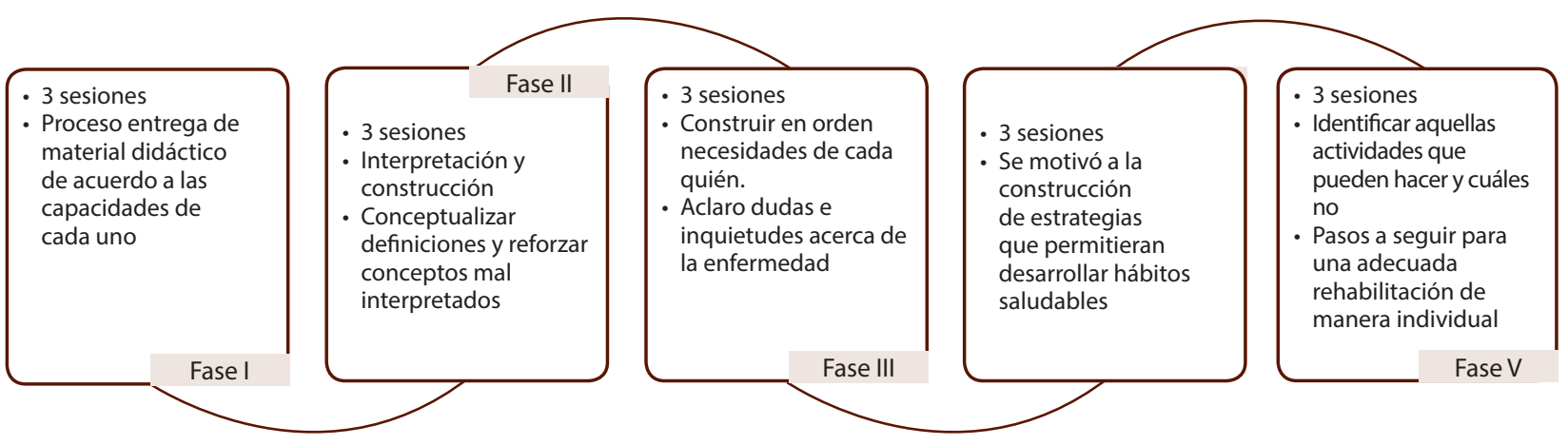

Figura 1: Diagrama de la intervención y mediación pedagógica aplicada a pacientes en proceso de rehabilitación cardiaca. 


\section{Fase de seguimiento}

Dos años después de realizado el proceso de mediación pedagógica, se realizó una labor de seguimiento y validación de las prácticas de vida saludable, a través de entrevistas abiertas al grupo de pacientes, con el propósito de indagar cómo el proceso de mediación pedagógica les había ayudado durante los dos años que estuvieron realizando la rehabilitación cardiaca por su propia cuenta.

\section{Técnicas e instrumentos para recolección de la información}

Para la recolección de la información requerida en el estudio, se implementaron tres estrategias que, desde el fundamento teórico del constructivismo, permiten diseñar una mediación pedagógica que promueve la construcción del nuevo conocimiento y refuerza el aprendizaje previo para que sea significativo a nivel individual.

La primera estrategia consistió en la construcción de los relatos de vida, cuyo propósito fue obtener una caracterización biográfica de cada paciente cardiaco. Esta técnica permite recolectar información sobre la vida del paciente. Mediante un conversatorio, el investigador instó a cada paciente a conversar sobre su patología, estado de salud, creencias, experiencias, entre otros aspectos, que considerara importantes. Los relatos o historias de vida se han consolidado como una de las fuentes más adecuadas para la obtención de datos, dentro de los documentos biográficos (Meneses y Cano, 2008).

La siguiente técnica de recolección de información fue el uso de frases incompletas, la cual ayudó a reforzar y complementar el relato de vida. Se construyó un instrumento de frases incompletas, el cual representó un conjunto de troncos verbales que la persona llenó, de acuerdo con lo primero que le vino a la mente cuando leyó las expresiones escritas por el mediador pedagógico. Este instrumento se debe realizar en forma cuidadosa y de acuerdo con los fines claramente definidos (Calzada, 2004). Con este instrumento se buscó comprobar el avance del aprendizaje que iba logrando cada paciente en la construcción de su propio conocimiento acerca de la alimentación, actividad física, relaciones sociales, manejo del estrés, depresión y ansiedad, además del conocimiento acerca de la enfermedad cardiaca. El instrumento de frases incompletas se utilizó con cada paciente al inicio, luego de dos semanas después de haber iniciado la intervención y al final de la mediación pedagógica, como medio de evaluación. Desde el constructivismo, este instrumento permitió conocer aspectos básicos relacionados con pensamientos, creencias y experiencias que la persona tenía con respecto a nutrición, enfermedad, actividad física, relaciones sociales, familiares, aspectos emocionales y afectivos. Además, con su aplicación a lo largo de la mediación pedagógica se pudo observar el avance que cada persona iba logrando, y desde su aplicación práctica y estos principios constructivistas permitió manifestar, de manera escrita, aquella información que fue relevante y significativa. 
doi: http://dx.doi.org/10.15359/ree.21-2.1

URL: http://www.una.ac.cr/educare

CORREO: educare@una.cr

Finalmente, se pasó el instrumento de libre asociación técnica, mediante el cual, a partir de la problemática que significa la patología cardiaca, se le presentaron, a cada paciente, conceptos que sirvieron de estímulo de pensamientos y sentimientos (Baumann, 1993) que, desde el constructivismo. Este permite observar cuán relevante y significativo fue para la persona un contenido que emergió a partir del nuevo conocimiento adquirido, ya que, con base en sus respuestas, queda evidenciada la modificación de la percepción de la persona y la relación que hace con respecto al concepto que el investigador escribió.

Esta información quedó a la libre, en el sentido de que cada quien, de forma positiva o negativa, emitía criterios desde su percepción. Este instrumento, al igual que el de frases incompletas, se pasó en tres diferentes momentos: al inicio, durante (dos semanas después de haber iniciado la intervención) y al final de la mediación pedagógica.

Al seruna investigación cualitativa, los instrumentos de naturaleza objetiva como encuestas impiden acercarse a lo que verdaderamente sienten y piensan las personas con problemas cardiovasculares. Los instrumentos se construyeron para cada situación en particular y han sido ampliamente escogidos como técnicas adecuadas en investigaciones fenomenológicas, hermenéuticas o interpretativas (Cornejo, Mendoza, Rojas, 2008).

\section{Criterio de validez}

Para efectos de esta investigación se utilizaron tres instrumentos diferentes para la recolección de datos (relatos de vida, frases incompletas y libre asociación), los cuales se administraron antes, durante y al final del proceso. La triangulación fue primordial para la contrastación de datos. Se obtuvo primeramente la información facilitada por todo el grupo participante del estudio, tanto al inicio de la mediación pedagógica, como al medio y al final. Además se contó con el análisis efectuado por el grupo de personal experto (validación de personas expertas); en este caso, los tres miembros del cuerpo asesor, quienes dieron su aporte profesional a la investigación de acuerdo con la información que se obtenía de los datos y, finalmente, se realizó el análisis según los fundamentos teóricos emergentes del marco teórico de la misma investigación; es decir, de los elementos teóricos. También se construyó un documento con expresiones realizadas y escritas por el grupo de pacientes con base en las categorías y subcategorías de análisis.

\section{Análisis de información}

Para el análisis de los resultados obtenidos en esta investigación se construyó un documento con expresiones realizadas y escritas por el grupo de pacientes con respecto a las categorías y subcategorías de análisis, a partir de las cuales se elaboró un texto que fue reforzado con referente 
científico, como sustento teórico del progreso que tienen las personas a partir del aprendizaje que van logrando con el nuevo conocimiento adquirido Para efectos de esta investigación se pretende que la persona con cardiopatía, al alcanzar un nuevo conocimiento, mejore su calidad de vida y adopte comportamientos saludables a partir de la comprensión integral en las áreas psico-socio-afectiva, de nutrición, social y de ejercicio físico, utilizando para la adquisición del nuevo conocimiento los principios y estrategias que propone el modelo constructivista.

\section{Resultados y análisis de interpretación}

Para identificar a los sujetos participantes y para efectos de protección de su identidad se utilizará la simbología "P1", “P2", P3, P4 y P5, para designarles. Las respuestas generadas por cada paciente se interpretaron de manera individual, en sus propios esquemas mentales; por tanto, este análisis está determinado por un proceso dinámico, interactivo a través del cual la información externa fue interpretada y reinterpretada por cada paciente cardiaco, quien iba construyendo progresivamente su conocimiento de acuerdo con su realidad. La organización de la información se basó en cinco grandes categorías, las cuales son fundamentales para el proceso exitoso de una rehabilitación cardiaca. La construcción de cada instrumento posterior fue estructurada teniendo en cuenta el avance que iban logrando, conforme adquirían nueva información acerca de la rehabilitación cardiaca a partir de sus pensamientos, inquietudes, así como de sus experiencias de vida. Para efectos de este artículo se presentarán algunas expresiones de manera textual, tal como las manifestó cada paciente, con el objetivo de evidenciar el avance que lograron al utilizar el constructivismo como herramienta para un mejor aprendizaje acerca de una adecuada rehabilitación cardiaca.

Para la categoría social-afectiva, uno de los principales factores emocionales en el paciente cardiaco, se manifestó el estrés, clasificado como uno de los factores de riesgo que favorece la aparición de trastornos cardiovasculares (Fernández, Bas-Sarmiento, González, Aranda y Martelo, 2004).

Para la mayoría de pacientes fue un factor difícil de manejar especialmente porque desconocían cómo hacerle frente a una situación estresante y lo asociaban al pensamiento de que el fallo cardiaco pudo ocasionar la muerte.

P1 “...yo tengo estrés, cualquier cosa me da estrés, pero, ¿cómo lo puedo controlar?, es que no he logrado hacerlo..."

Rodrigo:"...me cuesta manejar el estrés, tal vez cuando yo digo una cosa y me contradicen ahí me estreso rápido..."

Una vez finalizada la mediación pedagógica y con la construcción del conocimiento que cada quien iba ejecutando, mostraron los siguientes resultados: 
doi: http://dx.doi.org/10.15359/ree.21-2.1

URL: http://www.una.ac.cr/educare

CORREO: educare@una.cr

P1:“...Es muy importante platicar con él un día de estos, como son todos los psicólogos tienen un panorama general..."

“...me ayudó mucho porque me hizo analizar qué es lo que está pasando..."

P3:"...yo me he sentido bastante tranquilo y como que le hace falta a uno venir..."

Como se puede apreciar en las manifestaciones de la paciente y los pacientes que participaron en esta investigación, existió una evolución en cuanto a la forma de enfrentar las situaciones estresantes. Además de la información suministrada que facilitaba conocimientos de la enfermedad en la mediación pedagógica, se les ayudó a "aprender a aprender", es decir, cómo buscar información en caso de tener duda sobre algún aspecto relacionado con la rehabilitación cardiaca o las temáticas abordadas durante la investigación (Martínez, Rodríguez, Rodríguez y Rodríguez, 2005). En la categoría "nutricional" se reflejó un comportamiento característico del tipo de alimentación de las personas que han sufrido un evento cardiovascular. La alimentación de esta población, antes de sufrir el evento o antes del diagnóstico de sus problemas cardiovasculares era muy deficiente.

P4: “...un sábado comí y bebí como un cosaco para no perder la costumbre..."

P5: "...como pescado bastante, día de por medio..." / "...como bastante, aquí verduras, zanahoria, chayote, ayote, ensalada de remolacha con lechuga, pero me pasa que parece que eso no me sustenta..."

Finalizada la mediación pedagógica se mostraron los siguientes resultados:

P4: "...La comida de domingos, o sea la comida deliciosa es el arroz y los frijoles, y ojalá que los frijoles se vean cremoso, que no se vean como balines brillantes, brillantes, esos que se ven así como cremositos bien... eso es delicioso, lo que pasa es que son cantidades mínimas que podés comer..."

P5: “...yo eliminé la comida comía plátano arroz, frijoles, tallarines, guiso de pipíen..."

"...yo antes tenía que echarme otro plato de gallo pinto y ahora no, como que es psicológico..."

Como lo manifiesta Meza (2003), una apropiada alimentación se convierte en una necesidad básica de la persona y define su estado nutricional, por lo que representa un indicador de salud y de calidad de vida. Se aprecia un cambio importante en las expresiones del grupo de pacientes, 
algunos decidieron continuar con un menú para realizarlo todos los días, este aspecto resalta la autoorganización a la que llegó la mayoría de participantes de forma autónoma, es decir, fueron construyendo de manera individual la representación más sana de alimentarse. Carretero (1993) manifiesta que la mediación pedagógica constructivista debe ser una actividad que coadyuve en la construcción de un comportamiento caracterizado por hábitos de vida saludable, en donde prevalezca la salud integral como eje central de la rehabilitación cardiaca natural.

Para la categoría social, muchas personas experimentan estados de soledad y sentimientos de impotencia, de hecho, en ocasiones se sienten poco productivas. Encontrar apoyo social tanto del cónyuge, como de amistades, familiares y de personas con las condiciones similares beneficia su rehabilitación. Es indispensable conocer cuál ha sido el desenvolvimiento de estas personas antes del evento cardiaco y posteriormente a él en el área social.

P4: "...yo tengo 4 hijas que todas fueron casadas y un hijo varón, todos fueron casados, mis hijas mucho llegan a la casa como es mamá, verdad, entonces ellas quieren organizar mi vida..."

P2: “...Yo me casé en el 1972, pero seguí con mi ritmo de vida, pero mi señora que es una santa me soportó con mi ritmo de vida..."

Para el momento final de la intervención utilizando la mediación pedagógica, las siguientes fueron las expresiones de P4 y P2:

P4:“...se necesita gente para uno recuperarse y la parte muy importante la parte emocional, si uno no habla, no tiene con quien hablar, no tiene con quien expresarse, entonces para eso es el entorno familiar..."

P2: "...Mi hijo y mi nuera me ayudan mucho porque los dos son nutricionistas..."

El grupo de pacientes expresó la importancia que tiene la familia en el proceso de rehabilitación cardiaca. Las personas que participaron en la investigación asumieron un papel más activo, un mayor protagonismo y dinamismo, construyendo formas para abordar a sus familiares, de manera que sean parte efectiva en su recuperación. De acuerdo con García y Mas (s. f.), la persona debe desarrollarse en vinculación con otras, igual sucede con su recuperación, donde el acompañamiento puede convertirse en un factor fundamental para el éxito.

Para el comportamiento cognitivo, que representa una categoría enfocada en determinar cuánto aprendizaje había construido cada paciente con respecto a la enfermedad, sus características, consecuencias y posibles soluciones para su recuperación, los resultados de la evaluación inicial muestran lo siguiente: 
doi: http://dx.doi.org/10.15359/ree.21-2.1

URL: http://www.una.ac.cr/educare

CORREO: educare@una.cr

P1: “...Yo creo que los infartos dan por una suma de circunstancias, o sea a mí se me taponaron 5 venas de grasa, me imagino que debe haber sido por el acumulo de tanta cosa que he comido..."

P4: "...quien le ayuda a uno hacer un análisis correcto de la situación..."

P5:“...para serle sincera ni sabía qué era el infarto ni qué era peligroso al corazón y yo me asusté un poquito porque yo he oído decir que de un infarto se muere uno..."

Los resultados mostraron que la mayoría de los pacientes ignoraba cuáles eran las características de la enfermedad cardiaca, algunos consideraban que este problema le puede dar a cualquier persona; sin embargo, en algunos casos llegaron a la conclusión de que los malos hábitos de vida facilitaron el desarrollo de esta patología y que este problema podría incluso llevarles a la muerte. Esto es consistente con lo reportado por Albelo (2008), quien concluyó que gran cantidad de pacientes con problemas cardiovasculares desconocen sobre la cardiopatía isquémica antes de iniciar cualquier programa educativo. De acuerdo con Acosta, Debs, De la Noval, Dueñas (2005), las personas solo accederán a modificar su estilo de vida o a seguir un tratamiento, si son capaces de entender la magnitud del riesgo que significa no asumir un cambio.

La mediación pedagógica constructivista se constituyó en una convergencia de diversas aproximaciones didácticas, al partir del desarrollo psicológico del grupo de pacientes, particularmente desde el punto de vista de su formación intelectual y la intersección con los aprendizajes previos acerca de la enfermedad cardiaca (Díaz y Hernández, 1999). Una vez finalizada la mediación pedagógica con el enfoque constructivista, tanto su comportamiento como la comprensión acerca de lo que representa la enfermedad se modificó de manera representativa, lo cual se aprecia en las siguientes expresiones:

P1:“...En realidad el fin es ampliarle las arterias a uno para que tenga mejor oxigenación y que acumule menos ácido láctico en los músculos..."

P4: “...Para mí es tan importante el factor psicológico como el factor clínico, como la alimentación, como el ejercicio, yo no puedo tener una rehabilitación completa, una buena rehabilitación..."

P5: "... Muy bien porque he entendido un poco referente a la operación, antes tenía duda de que no sabía nada pues..." 
En los resultados mostrados para la subcategoría "conocimiento de la enfermedad", hubo una evolución importante de la paciente y los pacientes, en cuanto al grado o nivel de conocimiento acerca de la enfermedad cardiaca. Laham (2008) explicó que en el manejo educativo del paciente, se debe tener claro el hilo conductor, que consiste en pasar a la persona de pasiva, sin control sobre su vida; a ser una persona activa, que pueda controlar sus circunstancias, que sea quien plantee estrategias y desarrolle habilidades que le permitan interactuar y crecer en su entorno, y dar respuesta eficaz ante el proceso de rehabilitación cardiaca. Según Barrios (2008), en el constructivismo se desarrollan procesos mentales que permiten obtener conocimiento nuevo que, una vez procesado, se relaciona con aquellos conocimientos anteriores, lo cual permite que se adquiera una nueva competencia para aplicar lo nuevo conocido a una situación determinada. A su vez, Barrios (2008) consideró que la secuencia de asimilación-acomodación-aplicación es parte del aprendizaje personal y que en la educación llevada a cabo con personas adultas se convierte en una estrategia pertinente para lograr un cambio en su consciencia y comportamiento.

Para la subcategoría "conocimiento de la rehabilitación", los resultados mostraron diversas opiniones de los sujetos pacientes, de acuerdo con sus vivencias, así se encontraron comentarios acerca de cómo debería ser la rehabilitación cardiaca. Los siguientes se realizaron antes de realizar la mediación pedagógica:

P1: “...me interesa saber información de lo que estoy haciendo para ver si que puedo hacer más o que puedo hacer menos, o sea que esfuerzo puedo hacer y qué esfuerzo no puedo hacer, tomando decisiones propias..."

P4: "...voy a estar bien rehabilitado tanto emocionalmente como físicamente, porque si por mí fuera yo salgo de aquí y en la tarde hago otra hora de elíptica..."

P5:"...por eso estoy asistiendo aquí, porque él (médico) me dijo que tenía que reforzar un poquito el corazón, porque estaba muy débil..."

Los resultados de este primer momento mostraron que cada paciente tenía conocimiento limitado en cuanto al proceso de rehabilitación cardiaca. Alarcón, Cortés y Rodríguez (2006) argumentaron que el entendimiento de los estados emocionales y de las cogniciones es fundamental al iniciar con la recuperación de cada paciente cardiaco, ya que permite buena empatía y favorece, así, la explicación y comprensión de su recuperación física, emocional y social.

Posterior a la mediación pedagógica, los sujetos pacientes expresaron algunas inquietudes y fueron construyendo nuevo conocimiento durante el proceso de mediación pedagógica constructivista, con respecto a la forma de hacer el ejercicio y qué medios utilizar, la forma diferente de ver la rehabilitación cardiaca y el análisis autorreflexivo acerca de la evolución que tuvieron durante la intervención implementada. Estas son algunas de sus expresiones: 
doi: http://dx.doi.org/10.15359/ree.21-2.1

URL: http://www.una.ac.cr/educare

CORREO: educare@una.cr

P1: "...digo yo y debería ser obligatorio, no una decisión propia, sino que una cosa obligatoria, la fase 3 pues ya, ahí que no haya ningún control, pero pasar de la fase 1 a la fase 2 debería ser obligatorio..."

P4:"...el pecado más grande y creo que lo vamos a comentar por segunda vez, el hecho de que los hospitales no tengan sistemas de rehabilitación..."

P5: "...cuando vengo aquí a hacer ejercicio, yo me siento como limpia..."

Como se puede apreciar, la evolución que tuvo el grupo de pacientes cardiacos durante el proceso de mediación pedagógica constructivista, en las expresiones, evidenció que la comprensión era diferente con respecto a la visión que tenía acerca de la rehabilitación cardiaca, no solo de lo que significa un proceso de esta índole para cualquier paciente, sino que incluso hasta recomendaciones que algunos de ellos hicieron con respecto a la forma que debería ser la rehabilitación. En este sentido, García y Mas (s. f.) indicó que los pensamientos negativos, las autorreflexionesy el análisis permiten hacer cambios racionales que conviertenesquemas mentales anteriores en nuevos, por lo que cognitivamente se les asigna otro significado a las cosas. Brunner et al. (1966) expresaron que la condición primordial para aprender un nuevo conocimiento de manera significativa es, precisamente, tener la experiencia personal de descubrirla.

En el caso de la última categoría de análisis, la cual corresponde al conocimiento alcanzado acerca de lo que representa la actividad física, al inicio de la mediación pedagógica estas fueron sus expresiones de acuerdo con la comprensión que cada quien tenía hacia la práctica de actividad física:

P1: “...todos los días caminaba qué sé yo, 8 o 9 am hasta las 12 de la tarde..."

Rodrigo:"...Me gustaba jugar mejengas allá en las bananeras..."

P4: “...yo hacía ejercicio 5 veces por semana, no sé si había estado actuando mal y ahora solo 3 veces por semana y a niveles muy bajos..."

Los resultados obtenidos evidencian, en la subcategoría "actividad física", que mantenían algún nivel de actividad física, pero de forma básica y sin ningún principio científico, algunos pacientes refirieron que hacían ejercicio por recomendaciones médicas, sin ser el médico o la médica especialista para hacer ese tipo de recomendaciones, por lo que la persona inicia la actividad sin saber exactamente de qué forma hacerla o sin conocer en qué capacidad se encuentra su organismo para soportar una determinada intensidad de ejercicio. 
Durante la mediación pedagógica constructivista, se desarrollaron estrategias de aprendizaje con el fin de que tuvieran un conocimiento pertinente $y$, por lo tanto, significativo acerca de lo que representa la actividad física sistematizada o planificada y el conocimiento adecuado acerca del ejercicio físico, la actividad física y la influencia de ella en una rehabilitación cardiaca exitosa.

P1:"...bueno los ejercicios de ustedes deberían recomendarles a los cardiólogos y es por esto y por esto y por esto, así debería de funcionar, debería ser valer, su idea, su teoría, su propuesta me parece bien encaminada y excelente..."

P3: "...comenzar con 10 minutos en una bicicleta de esas y veo que me está afectando algo pararía en ese momento, inmediatamente, haría variaciones vea, un día tal vez 10 minutos, tal vez el otro día lo agarraría como hacen aquí día de por medio, 20 minutos, depende de lo que yo vaya a aguantar..."

P4:“...una hora 3 veces a la semana, o sea yo me he venido excediendo, que porcentaje más alto..."

Se encontró que los sujetos pacientes tuvieron una evolución en cuanto a la modificación de su comprensión y grado de conocimiento para esta subcategoría de análisis. Las expresiones encontradas para el momento final de la intervención pedagógica constructivista mostraron que durante el proceso mejoraron su nivel de conocimiento, producto de la construcción significativa en el aprendizaje acerca de la actividad física planificada. La autorreflexión realizada les permitió un análisis crítico acerca de cómo especialistas en medicina sin capacitación no pueden prescribir actividad física planificada, ya que desconocen sobre esa área en particular.

A lo largo del proceso guiado, los sujetos pacientes modificaron su nivel de comprensión, lo que les ayudó a asimilar, de forma significativa, la manera de iniciar la rehabilitación cardiaca mediante el ejercicio. Tanto en el aprendizaje significativo como en la mediación pedagógica, el material o el rol que cumple el mediador o mediadora debe ser potencialmente significativo y debe tener una relación con las estructuras de conocimiento de las personas, ya que esto facilita que el conocimiento sea asimilado y difícil de olvidar a largo plazo (Serrano y Pons, 2011). De acuerdo con Villavicencio (2004), es importante que la persona elabore estrategias de autoplanificación, es decir, que reflexione sobre planes de acciones realistas y efectivas; esto permite una mejor construcción del aprendizaje, dándole un carácter significativo.

Una vez finalizada la investigación, se procedió a realizar un seguimiento dos años después de haber recibido la intervención pedagógica y estos fueron algunos de los comentarios obtenidos posterior a la entrevista: 
doi: http://dx.doi.org/10.15359/ree.21-2.1

URL: http://www.una.ac.cr/educare

CORREO: educare@una.cr

\section{Categoría nutricional}

P1:“...bajé el azúcar, bajé la ingesta de harina como a la mitad, estoy comiendo muchas frutas, muchas ensaladas y muchos vegetales, disminuir la ingesta de arroz, la sal por el asunto de la presión arterial..."

P2: "...Llevo un control y tomé la decisión de hacerme controles del perfil lípido, con lo aprendido ahora como muchas frutas, eliminé grasas, aumentemos el consumo de ensaladas y verduras..."

P3:“...En realidad evito comer carnes rojas, grasas empecéa consumir mucha fruta, vegetales..."

P4:"...pero Jorge tengo muy claro cuáles alimentos me benefician, quécambios debo de hacer..."

\section{Categoría actividad física}

P1:"...hago todos los días 30 minutos caminando y bicicleta 15 como lo aprendí en el proceso educativo..."'... me voy controlando las pulsaciones entre 120 y 130 latidos con el reloj polar..."

P2: "...Desde que salí del programa trato de caminar, pero ahora lo sustituyo con el gimnasio hago banda sin fin...", "...siempre me controlo con el monitor cardiaco polar..."

P3: "...Salgo a caminar de 3 a 4 por que aprendí que era de día por medio...," "...además me controlo con la frecuencia cardiaca, el reloj que aprendí debía comprar..."

\section{Categoría emocional-afectivo}

P1: “...pero la estrategia que implemente de conversar con familiares me permite hoy sentirme con más calma..."

P2: “...He manejado el estrés y depresión bastante bien, el proceso educativo me ayudó infinitamente, no experimento ningún tipo de ansiedad..."

P3: "...Lo que he hecho es disimular que tengo el problema, si de repente siento alguna molestia ya no me estreso, como lo aprendí..."

P4:“...En estos dos años no me ha afectado nada, más bien he duplicado el trabajo y ha sido placentero..." 


\section{Categoría social}

P1:"...yo les digo a mis amigos y familiares que del corazón no me voy a morir, he sentido un apoyo muy grande de la familia ..."

P2: "...por ejemplo en las reuniones con amigos yo antes consumía alcohol, ahora más bien lo trato de evitar, aunque ellos me motivan, yo prefiero beber algún refresco ..."

P3:“...Toda la dinámica de las relaciones sociales ha sido muy buena, en la familia ya casi no se conversa de la enfermedad ..."

\section{Categoría conocimiento general}

P1: “...En este momento por el conocimiento alcanzado incluso estoy dando recomendaciones a mis hijos y familiares acerca de los cuidados preventivos..."

P2: "...Si una persona tuviera el problema que yo tuve le diría es que trate de llevar la rehabilitación cardiaca y sobre todo el proceso educativo porque esto le va a dar el conocimiento necesario para seguirse cuidando..."

P3:“...Con lo aprendido yo le podría decir a una persona que primero se ponga en manos de Dios, que tenga mucha fe y después qué cosas evitar por ejemplo la alimentación, cómo hacer el ejercicio ..."

P4: "...Si una persona estuviera con la misma situación que la mía yo lo primero que le recomendaría es que se eduque sobre hábitos sanos, como me sirvió a mí..."

Esposo de P5:"...Yo pienso que ella ya no siente el estrés que sentía antes y se siente muy segura de hecho vea que se fue hasta Nicaragua ..."

Finalmente, se les realizó la consulta acerca de cómo la mediación pedagógica había ayudado en su proceso de rehabilitación y las expresiones finales fueron las siguientes:

P1:“...Me dio una guía en la vida, yo me fui del proyecto de rehabilitación cardiaca por la distancia que quedaba el lugar y por las presas en la calle. Y tomé la decisión de que con lo aprendido yo podría hacer las cosas, el proceso educativo me cambió la visión y me generó insumos de cómo controlar el estrés, cómo realizar el ejercicio físico..." 
doi: http://dx.doi.org/10.15359/ree.21-2.1

URL: http://www.una.ac.cr/educare

CORREO: educare@una.cr

P2:"...Fue una experiencia muy satisfactoria, me hace estar muy tranquilo y me hizo tener un control sobre el ejercicio, alimentación y conocimiento general de la enfermedad..."

P3:“...El proceso de educación me dio la seguridad de que, si tengo que realizarme una intervención quirúrgica iría sin ningún tipo de temor. Además, el conocimiento para después de la operación mantenerme debidamente rehabilitado..."

P4:"...La información la tengo totalmente clara como para que si alguna persona sufre un problema de este tipo yo poder ayudarle a lo que debe hacer y además evidentemente aplicarla en mí..."

Esposo de P5: "...A ella le sirvió en todo, cambio en todo, antes todo le estorbaba, no comía por temor que lo que comiera le afectara el corazón, no se movía mucho por temor de que el corazón no le aguantara y ahora sale sola y camina largas distancias sin ningún tipo de temor..."

Como se puedeapreciar, la mediación pedagógica dos años después, los sujetos participantes de este estudio evidenciaron que lo aprendido producto de la propia construcción del nuevo conocimiento acerca de lo que conlleva un adecuado proceso de rehabilitación cardiaca fue significativo, ya que todavía se mantienen aplicando las estrategias de la mediación pedagógica.

\section{Conclusiones}

Lo trascendental de este proceso de investigación, en definitiva, es que permitió aclarar la pertinencia de una guía para pacientes cardiópatas en la construcción de conocimiento nuevo alrededor de la patología, como base para inducir a modificaciones en el comportamiento, que les ayuden en el proceso de rehabilitación cardiaca.

Cabe señalar que el proceso vivido fue una oportunidad para observar la forma de pensar y sentir de pacientes cardiópatas. De acuerdo con esos criterios, se logró comprender que el abordaje educativo debe ser personalizado, ya que cada quien tiene una forma diferente de comprender su patología y su realidad, en el contexto de sus experiencias previas, sus capacidades de aprendizaje y al acervo afectivo.

El hecho de compartir experiencias con las personas y observar su desenvolvimiento de acuerdo con cómo aprenden, permitió concluir que además de la construcción del nuevo conocimiento, es posible que este se mantenga aún dos años después de realizada la intervención, lo cual se evidenció a través de una entrevista semiestructurada. Esto indica que la persona con enfermedad cardiovascular debe poner el nuevo conocimiento aprendido

\begin{tabular}{l|l}
\hline 22 & Jorge Salas-Cabrera
\end{tabular}

Los artículos de la Revista Electrónica Educare del Centro de Investigación y Docencia en Educación de la Universidad Nacional, Costa Rica, se comparten bajo términos de la Licencia Creative Commons: Reconocimiento, No Comercial, Sin Obra Derivada 3.0 Costa Rica. Las autorizaciones adicionales a las aquí delimitadas se pueden obtener en el correo: educare@una.cr 
en práctica constante, de esta manera la información recibida puede llegar a ser significativa de forma específica; es decir, en la medida que los sujetos pacientes lleven a cabo prácticas diarias en su autocuidado, va a garantizar que el conocimiento aprendido no sea olvidado en el transcurso de los años. De hecho, esta es una de las consecuencias diarias que se perciben en las personas que han sufrido un problema cardiaco y que no han sido abordadas de esta manera, ya que al dejar de estar en contacto diario con su especialista, vuelven a hábitos de vida poco saludables, lo cual aumenta la posibilidad de que ocurra un nuevo evento cardiaco.

\section{Referencias}

Acosta, M., Debs, G., De la Noval, R. y Dueñas, A. (2005). Conocimientos, creencias y prácticas en pacientes hipertensos, relacionados con su adherencia terapéutica. Revista Cubana Enfermería, 21(3), 1-8. Recuperado de http://scielo.sld.cu/pdf/enf/v21n3/enf08305.pdf

Alarcón, D., Cortés, A. y Rodríguez, V. (2006). Competencias cognitivas, evaluación constructivista y educación inicial. Chile: Bravo y Allende Editores.

Albelo, M. (2008). Programa de intervención educativa para incrementar nivel de conocimientos sobre cardiopatía isquémica en pacientes del municipio Venezuela. MediCiego, 14(2) Recuperado de http://www.bvs.sld.cu/revistas/mciego/vol14 02 08/articulos/a7 v14 0208.htm

Álvarez, M. I., De la Rosa, T., Acosta, M. E., Albar, M. J., García, M. J. (2005). Evaluación del impacto del programa de rehabilitación cardíaca del hospital universitario Virgen Macarena en la calidad de vida de sus usuarios. Trabajo presentado en el $5^{\text {to }}$ Congreso Internacional de Cardiología. Recuperado de www.fac.org.ar/qcvc/llave/tl007e/tl007e.pdf

Ausubel, D. P., Novak, J. y Hanesian, H. (1983). Psicología educativa: Un punto de vista cognoscitivo ( $2^{\mathrm{a}}$ ed.). México: Trillas.

Baumann, S. (1993). Psychologie im sport. Aachen, Alemania: Meyer \& Meyer.

Barrios, C. (2008). Aplicación del enfoque constructivista a la educación sanitaria. Delos: Desarrollo Local Sostenible, 1(2), 1-13. Recuperado de http://www.eumed.net/rev/delos/02/cbn.pdf

Bravo, L. (2002). Psicología de las dificultades del aprendizaje escolar (7a ed.). Santiago de Chile: Editorial Universitaria.

Bruner, J. S. (1988). Desarrollo cognitivo y educación. Madrid: Morata.

Bruner, J. S., Olver, R. R. y Greenfield, P. M. (1966). Studies in cognitive growth. NewYork: Wiley. 
doi: http://dx.doi.org/10.15359/ree.21-2.1

URL: http://www.una.ac.cr/educare

CORREO: educare@una.cr

Cabrera, R., Motta, I., Rodríguez, C. y Velázquez, D. (2009). Nivel de conocimiento sobre autocuidado en la prevención de complicaciones diabéticas en ususarios del policíclinico Chincha. Revista Enfermeria Herediana, 3(1), 29-36.

Calzada, J. G. (2004). La técnica de las frases incompletas: Revisión, usos y aplicaciones en procesos de orientación vocacional. Recuperado de http://www.psi.uba.ar/academica/ carrerasdegrado/psicologia/sitios catedras/obligatorias/042 ttedm2c2/material/fichas/ tecnica de las frases incompletas.pdf

Carretero, M. (1993). Constructivismo y educación. Zaragoza: Edelvives.

Castro, R. (2004). Un modelo constructivista para la comunicación en la enseñanza de la matemática. Educere, 8(24), 119-127. Recuperado de http://www.redalyc.org/articulo. oa? id $=35602418$

Cerezo, P. (2008). Modelos de aprendizajes aptos para adultos. Recuperado de http://www.a3net. net/formador/doc/modelosaprendizaje.pdf

Coronel, M. y Curotto, M. (2008). La resolución de problemas como estrategia de enseñanza y aprendizaje. Revista Electrónica de Enseñanza de las Ciencias, 7(2), 463-479. Recuperado de http://reec.uvigo.es/volumenes/volumen7/ART11 Vol7 N2.pdf

Cornejo, M., Mendoza, F. y Rojas, R. C. (2008). La investigación con relatos de vida: Pistas y opciones del diseño del trabajo metodológico. PSYKHE, 17(1), 29-39. doi: https://doi. org/10.4067/S0718-22282008000100004

Cossette, S., Frasure-Smith, N. y Lespérance, F. (2001). Clinical implications of a reduction in psychological distress on cardiac prognosis in patients participating in a psychosocial intervention program. Psychosomatic Medicine, 63(2), p. 257-266. doi: https://doi. org/10.1097/00006842-200103000-00009

Cubero, F. (2009). Principios constructivistas y diversidad de la comunidad del aula. Recuperdo de http://www.redires.net/?q=node/378

Cuenca, N. (2007). Didáctica constructivista como práctica mediadora para una cultura de paz. REDHECS, 2(3), 15-30. Recuperado de http://publicaciones.urbe.edu/index.php/REDHECS/ article/view/404/975

Díaz, F., Hernández, G. (1999). Constructivismo y aprendizaje significativo (Capítulo 2). En F. Díaz y G. Hernández (Autores), Estrategias docentes para un aprendizaje significativo. Una interpretación constructivista (pp. 13-19). México-McGraw-Hill. Recuperado de http://www.ict.edu.mx/acervo educacion Constructivismo\%20y\%20aprendizaje $\% 20$ significativo F\%20Diaz.pdf 
Del Río, G., Turro, E., Mesa, L. D., Mesa, R. M. y De Dios, J. A. (2005). Protocolos y fases de la rehabilitación cardíaca. Orientaciones actuales. Medisan, 9(1), 1-7. Recuperado de http:// bvs.sld.cu/revistas/san/vol9 1 05/san14105.pdf

Esquivias, M. T., González, A. y Muria, I. (2003). Solución de problemas: Estudio evaluativo de tres enfoques pedagógicos en las escuelas mexicanas. Revista de investigación psicoeducativa y psicopedagógica, 1(2) 1-18. Recuperado de http://investigacion-psicopedagogica.org/ revista/articulos/2/espannol/Art 2 18.pdf

Escobar, J. (2003). Intervención pedagógica y didáctica en adultos y adultos mayores para la actividad física. Efdeportes.com, 9(63), Recuperado de http://www.efdeportes.com/efd63/ adultos.htm

Fernández, M., Bas-Sarmiento, P., Gonzáles, R., Aranda, J. y Martelo, M. Á. (2004). Estrés y enfermedad cardiovascular. Un abordaje conductual para la prevención. Metas de enfermería, 7(2), 14-18.

Gallego, D. J. y Ongallo, C. (2004). Conocimiento y gestión. Madrid: Pearsons Educación.

García, A. M. y Mas, K. (s. f.). Factores psicosociales que inciden en la rehabilitación del enfermo cardiaco. Recuperado de http://www.monografias.com/trabajos5/enfcard/enfcard2.shtml

Hernández, A. (2007). Atención psicoeducativa social en la escuela hospitalaria. Una experiencia significativa. En I Jornada nacional de pedagogía hospitalaria en Venezuela: La educacion prioridad de vida (pp. 1-14). Estado de Miranda, Venezuela. Recuperado de http://www. pedagogiahospitalaria.net/jornadas/2007/ponencias/Adelina Hernandez.pdf

Hernández, S. (2008) El modelo constructivista con las nuevas tecnologías: Aplicado al proceso de aprendizaje. Revista de Universidad y Sociedad del Conocimiento, 5(2) 26-35. Recuperado de http://www.uoc.edu/rusc/5/2/dt/esp/hernandez.pdf

Hernández, D. y Arranz, H. (2009). Prevención y rehabilitación cardiaca. Generalidades. En M. T. Portuondo, T. Martínez, J. Delgado, P. García, D. Gil, J. A. Mora, M. E. Vivas (Eds.), Manual de enfermería. Prevención y rehabilitación cardiaca (pp. 21-36). Recuperado de https://www. enfermeriaencardiologia.com/wp-content/uploads/cap 01.pdf

Ibáñez, C. (2007). Un análisis crítico del modelo triángulo pedagógico. Una propuesta alternativa. RMIE, 12(32), 435-456. Recuperado de http://www.redalyc.org/articulo.oa?id=14003220

Kunoichisama, K. (s. f.). El constructivismo en el aula. Valparaíso: UPLACED. Recuperado de http:// www.monografias.com/trabajos35/constructivismo-aula/constructivismo-aula.shtml 
doi: http://dx.doi.org/10.15359/ree.21-2.1

URL: http://www.una.ac.cr/educare

CORREO: educare@una.cr

Laham, M. (2008). Psicocardiología: Su importancia en la prevención y la rehabilitación coronarias. Suma Psicología, 15(1), 143-170. Recuperado de http://www.redalyc.org/ articulo.oa?id=134212604006

Martínez, A. A., Rodríguez, A., Rodríguez, C. y Rodríguez, A. (2005). Percepción de las estrategias de afrontamiento ante el estrés en pacientes ingresados en una unidad de coronarias. Enfermería en Cardilogía, 12(34), 15-21. Recuperado de https://www. enfermeriaencardiologia.com/wp-content/uploads/3401.pdf

Meneses, M. T. y Cano, A. (2008).Técnicas conversacionales para la recogida de datos en investigación cualitativa: La historia de vida (I). Nure Investigación, 37, 1-6. Recuperado de http://www.nureinvestigacion.es/OJS/index.php/nure/article/view/424/415

Meza, N. (2003). La revisión de los hábitos alimentarios de la población costarricense. En A. Blanco (Comp.), Memoria Seminario índice glicémico en salud y alimentación humana. Tres Ríos, Costa Rica: INCIENSA.

Mora, J. A. (2009). Programas de prevención y rehabilitación cardiaca. En M. T. Portuondo, T. Martínez, J. Delgado, P. García, D. Gil, J. A. Mora, ... M. E. Vivas (Eds.), Manual de enfermería. Prevención y rehabilitación cardiaca (pp. 123-188). Recuperado de https://www. enfermeriaencardiologia.com/wp-content/uploads/cap 03.pdf

Moreno, S. M. (2010). Educación para adultos (Tesis de grado). Universidad Militar Nueva Granada. Recuperado de http://repository.unimilitar.edu.co/bitstream/10654/369/1/ MorenoZarateSandra2010.pdf

Núñez, J. C., Solano, P., González-Pienda, J. A. y Rosário, P. (2006). El aprendizaje autorregulado como medio y meta de la educación. Papeles del Psicólogo 27(3), 139-146. Recuperdo de http://www.redalyc.org/pdf/778/77827303.pdf

Palacios, M. A. (2000). La educación en América Latina y el Caribe. Los procesos pedagógicos. En Unesco (Ed.), Seminario de análisis prospectivo de la educación en la región de América Latina yel Caribe (pp. 361-381). Santiago: Unesco. Recuperado http://unesdoc.unesco.org/ images/0013/001349/134963s.pdf

Perera, H. (2009). [Reseña del libro El aprendizaje autónomo en la educación superior, por J. Rué]. Revista española de pedagogía, 2(1), 125-127. Recuperado de http://intercambios. cse.edu.uy/wp-content/uploads/2014/05/art12 resena1_perera.pdf

Restrepo, B. (2005). Aprendizaje basado en problemas (ABP). Una innovación didáctica para la enseñanza universitaria. Educación y Educadores, 8, 9-19. Recuperado de http://www. redalyc.org/articulo.oa?id=83400803 
Ruiz, F. J. (2007). Modelos didácticos para la enseñanza de las ciencias naturales. Revista Latinoamericana de Estudios Educativos, 3(2), 41-60. Recuperado de http://www.redalyc. org/articulo.oa?id=134112600004

Salinas, B., Huertas, M. G., Porras, L. H., Amador, S. E. y Ramos, J. M. (2006). Uso significativo en la educación en adultos en el medio rural. Resultados de la aplicación piloto de un modelo. Revista Mexicana de Investigación Educativa, 11(28), 31-60. Recuperado de http://www. redalyc.org/pdf/140/14002804.pdf

Sánchez, I., Moreira, M. A.y Caballero, C. (2011). Implementación de una renovación metodológica para un aprendizaje significativo en Física I. Latin-American Journal of Physics Education, 5(2), 475-484. Recuperado de http://www.lajpe.org/june11/26 LAJPE 521 Ivan Sanchez preprint corr f.pdf

Serrano, J. M. y Pons, R. M. (2011). El constructivismo hoy: Enfoques constructivistas en la educación. Revista Electrónica de Investigación Educativa, 13(1), 1-27. Recuperado de http:// www.scielo.org.mx/pdf/redie/v13n1/v13n1a1.pdf

Soler, E. (2006). Constructivismo, innovación y enseñanza afectiva. Caracas: Editorial Equinoccio.

Sosa-Simón, C. A., Pavia-Carillo, E., Mendoza-Cruz, J.U. (2009). Aptitud de enfermería en pacientes con paro cardiorrespiratorio: Alcances de una estrategia educativa. Revista de Enfermeria Instituto Mexicano del Seguro Social, 17(2), 85-90. Recuperado de http://www.medigraphic. com/pdfs/enfermeriaimss/eim-2009/eim092e.pdf

Torres, M. J. (2008). Procedimiento didáctico en el proceso pedagógico para fortalecer el aprendizaje cooperativo de los estudiantes de 8 años deeducación básica del colegio (Tesis de licenciatura). Universidad Estatal de Bolívar, Venezuela.

Villavicencio, L. M. (2004). El aprendizaje autónomo en la educación a distancia. En Primer Congreso Virtual Latinoamericano de Educación a Distancia (pp. 1-11). Pontificia Universidad Católica del Perú. Recuperado de http://geoservice.igac.gov.co/moodle/file.php/1/moddata/ glossary/5/32/El aprendizaje autonomo en educacion a distancia.pdf

Vygotski, L. (1978). Mind in society. The development of higher psychological processes. Cambridge: Harvard University Press. 